qui peut amener des résultats incertains surtout pour des fromages vieux.

SöNCKE-KNUDSEN a démontré que les électrodes à la quinhydrone et à l'hydrogène, préparées avec un mélange de 1 partie de fromage et de 2 parties d'eau, puis élimination par centrifugation de la partie insol ble, donnent des différences de potentiel calculées. Comme nos électrodes $\mathrm{H}_{41}$ et $\mathrm{H}_{42}$ doivent être de la même composition, il m'a paru inutile d'exécuter des mesures analogues à celles de KNODSEN.

\title{
SUR QUELQUES COIMPOSANTS ET PROPRIÉTÉS DU LAIT DES VACHES CASTRÉES
}

\author{
par le Prof. Dr A. STAFFE,
}

Directeur de l'Institut de Laiterie et de Bactériologie agricole de l'Ecole supérieure pour la eulture du sol, à Vienne,

avec la

collaboration de MM. A. JANOSCHEK et W. SCHEIMPFLUG.

\section{Introduttion.}

La castration ou ovariotomie des vaches a déjà été pratiquée au milieu du xvIII siècle, en Saxe et en Suède, ainsi que le rapportent Frenzel et Retzius $(4,20)$, et au xix ${ }^{e}$ siècle, à l'instigation d'un agriculteur du Nord de l'Amérique, spécialement en France puis en Allemagne, Autriche et en Suisse. Cette opération, pratiquée par un vétérinaire habile, est actuellement presque sans danger. Elle est recommandée contre la nymphomanie des vaches taurelières ou chez les vaches qui, pour une raison quelconque, ne sont plus utilisées pour la reproduction, pour remédier à l'influence gênante des "chaleurs", de la gestation et du vêlage sur la lactation.

La castration pratiquée au début d'une période de lactation provoque un arrêt de la lactation, mais, d'après certains observateurs (17), on observe aussi une augmentation de rendement laitier, une amélioration du goût du lait (surtout chez les vaches nymphomanes), ainsi qu'une augmentation du taux en lactose, matière grasse et ca séine.

Les observations des différents chercheurs $(2,6,7,13,15,19,21$, 22) sur l'influence de la castration et ses conséquences sur les composants cités ci-dessus et les propriétés du lait ne sont pas concordantes ; pour cette raison, la présente contribution tendant à éclaircir ce problème peut présenter quelque intérêt.

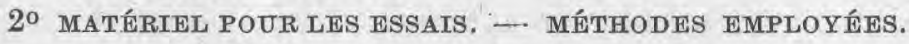

Les présentes recherches ont été provoquées par le fait qu'à fin 
septembre 1928, la Société viennoise d'exploitation agricole du domaine de Laxenbourg fit l'acquisition d'un lot de bétail du Tyrol comprenant 32 vaches de montagne des vallées supérieures de l'Inn. Les 21-22 janvier 1929, 8 vaches furent castrées. Toutes ces vaches avaient vêlé depuis le 15 octobre jusqu'au 15 décembre 1928 ; elles se trouvaient done au début de ces recherches (janvier 1929), dans le premier stade de lactation. Une des vaches castrées fut abattue d'urgence pour une raison pathologique le 2 mai 1929, une deuxième fut éliminée, le 2 décembre 1929, car elle ne donnait plus que 6 litres et demi de lait par jour.

On a déterminé : le taux en matière grasse, le poids spécifique, le lactose selon KoLthofr (8), le chlore selon KoranYi-ZaribNICKY (24b), le point de congélation, l'azote total, la caséine, l'albumine et la globuline, le reste d'azote selon ZARIBNICKY-MUNCHBERG, modi-

TABLEAU I.

Quantité de lait en litres.

\begin{tabular}{|c|c|c|c|c|c|c|}
\hline Date & $N^{\circ} 406$ & No 408 & $N^{\circ} 410$ & No 413 & $N \cdot 420$ & No 426 \\
\hline $8-1-29$ & 11.75 & 13.25 & 13.50 & 8.50 & 15.75 & 8.25 \\
\hline $1-2-29$ & 15.75 & 16. - & 14.50 & 11.- & $18 .-$ & 9.75 \\
\hline $19-2-29$ & 14.25 & 14.50 & 12.50 & $10 .-$ & 16.- & 10.- \\
\hline $5-3-29$ & $15 .-$ & 16.- & 14.- & 11.- & 17.50 & 10.25 \\
\hline $19-3-29$ & 14.25 & $14 .-$ & 12.- & 9.25 & 15.50 & 9.25 \\
\hline $4-4-29$ & 16.50 & 16.50 & 13.50 & 10.25 & 17.- & 10.25 \\
\hline $18-4-29$ & 15.50 & 14.25 & 12.50 & 9.25 & $15 .-$ & 10. - \\
\hline $3-5-29$ & $15 .-$ & 14.25 & 12.75 & 9.50 & 15.25 & 9.75 \\
\hline $17-5-29$ & 14.75 & 11.50 & 11.75 & 9.50 & 15.75 & 10.25 \\
\hline $6-6-29$ & 17.25 & 14.50 & 14.50 & 10.- & 14.25 & 10.- \\
\hline $15-6-29$ & 17.- & 14.50 & 14.- & $10 .-$ & $14 .-$ & $10 .-$ \\
\hline $1-7-29$ & 15.75 & 13.25 & 12.50 & 8.50 & 10.- & 11.- \\
\hline $15-7-29$ & 15.- & 13. - & 13.75 & 8.75 & 12.- - & 11. - \\
\hline $1-8-29$ & $14 .-$ & 13.25 & 13.75 & 8.50 & 12.50 & 11.75 \\
\hline $15-8-29$ & 14.50 & 12.50 & 10.75 & 8.75 & 14.50 & 9.50 \\
\hline $5-9-29$ & 14.75 & 13.50 & 11.75 & 9.- & 13.50 & 9.75 \\
\hline $17-9-29$ & $15 .-$ & $14 .-$ & 11.75 & 9.25 & 14. - & 9.75 \\
\hline $1-10-29$ & $12: 75$ & $12 .-$ & 11.75 & 9.75 & 12.50 & 9. - \\
\hline $15-10-29$ & 11.50 & 11.75 & 10.50 & 8.25 & 11.50 & 8.75 \\
\hline $4-11-29$ & 12.75 & 11.50 & 10.25 & 9.25 & 11.75 & 8.50 \\
\hline $15-11-29$ & 11.75 & 9.50 & 9.75 & 9.25 & 11.50 & 8.- \\
\hline $3-12-29$ & 11.25 & 10.50 & 9.75 & 9.- & 11.75 & 8.75 \\
\hline $16-12-29$ & 11.- & 10.25 & 10.25 & 8.- & 12.75 & 8.50 \\
\hline $2-1-30$ & 8.75 & 9.75 & 9.75 & 8.75 & 11.50 & 8.25 \\
\hline $15-1-30$ & 8.25 & 9.- & 8.75 & 9.- & 12.- & 8.50 \\
\hline $3-2-30$ & 8.75 & 11.50 & 11.25 & 8.75 & 12.25 & 9.50 \\
\hline
\end{tabular}


B. Vaches témoins.

\begin{tabular}{|c|c|c|c|c|c|c|c|c|c|c|}
\hline Date & 401 & 402 & $N^{\circ} 404$ & $\mathrm{~N} \circ 409$ & 415 & 425 & $z 4]^{1}$ & 427 & $\left.8\right|^{5}$ & \\
\hline $8-1-29$ & 11. & 16.50 & 11.25 & 11.75 & 12.- & 11.25 & 10.75 & 15.75 & 11.50 & 10.50 \\
\hline $1-2-29$ & 10.75 & 19.75 & 11.25 & 12.25 & 13.25 & 12.- & 11.25 & 18.25 & 13.50 & 11.50 \\
\hline $19-2-29$ & 8.75 & 18.- & 10.75 & 11.75 & $12 .-$ & 11.75 & 9.75 & 17.75 & 13.25 & 10.75 \\
\hline $5-3-29$ & 75 & 17.- & 10.50 & 12.- & 11.25 & 12.75 & 10.75 & 16.75 & 12.75 & 10.00 \\
\hline $19-3-29$ & .25 & 15.75 & $10 .-$ & 11.25 & 11. - & $12 .-$ & 10.75 & 16.25 & 11.50 & 10.75 \\
\hline $4-4-2$ & .25 & 19.- & 11.25 & 13.50 & 12.25 & $14 .-$ & 11.- & 16.50 & 13.75 & 11.75 \\
\hline $18-4-29$ & 25 & $15 .-$ & 11.25 & 12.25 & 10,75 & 14.- & 11.50 & 16.25 & 13.- & 11.25 \\
\hline $3-5-29$ & 75 & 15.75 & 10.75 & 13.25 & 11.25 & 13.25 & 11.- & 15.75 & 9.25 & 10.50 \\
\hline $17-5-29$ & 25 & 15.50 & 11.50 & 14.50 & 11.25 & 13.75 & $12 .-$ & $16,-$ & 10.25 & 11.75 \\
\hline $6-6-29$ & - & 16.25 & 12.50 & 15.75 & 9.50 & 15.50 & 12.50 & $16 .-$ & 11. - & 10.75 \\
\hline $15-6-29$ & . & 16.50 & 12.50 & 15.50 & 9.- & 15.25 & 12. - & 16.- & 10.50 & 10.25 \\
\hline $1-7-29$ & 11.25 & $14 .-$ & 11.50 & 14. & 9.25 & 13.25 & $12 .-$ & 9.50 & 11.- & $11 .-$ \\
\hline $15-7-29$ & 10.- & 13.- & 11.50 & $13 .-$ & 9. - & 13.- & 11.- & 16. - & 11. - & . \\
\hline $1-8-29$ & 9.50 & 12. - & 11.25 & 13.25 & 9.50 & 12.50 & $10 .-$ & 16.50 & 10.50 & $11 .-$ \\
\hline $15-8-29$ & 10.- & 13. - & 10.- & 13.50 & 9.50 & 11.50 & 9.50 & $15 .-$ & $10 .-$ & 10.75 \\
\hline $5-9-2$ & 8.50 & 11.- & 7.50 & 12.50 & 8.25 & 12.50 & 8.50 & 14.25 & 11.25 & 10.- \\
\hline $17-9-2$ & 8.50 & $13 .-$ & 8.50 & 13.25 & 8.- & 13.25 & 9.50 & 15.50 & $12 .-$ & 10.- \\
\hline 1 & 3.75 & 13.- & 9.75 & $13 .-$ & 10.- & 11.25 & 9.25 & 14.50 & 12.50 & 9.25 \\
\hline 15 & 8.- & 11.50 & 9.- & 12.- & 9.25 & 11.- & 8.25 & 13.50 & 10.75 & 9.5 \\
\hline 4-11 & 3.75 & 12.25 & 8.75 & 11.- & 8.25 & 10.75 & 9. - & 11.- & 11.50 & 9.75 \\
\hline $15-11-2$ & 7.75 & 10.25 & 9.25 & 9.75 & 9.50 & 10.50 & 9.- & 13.- & 11.- & 10.- \\
\hline 2 & 7.25 & 10.50 & 9.50 & 10.75 & 7.- & 11.75 & 6.50 & 8.50 & 0.50 & 10.- \\
\hline $16-12-2$ & 8.25 & 11.75 & $10 .-$ & 9.75 & 8.50 & 10.25 & 9. - & 12.75 & 10.75 & 9.2 \\
\hline $2-1-30$ & 9.- & 11.50 & 8.75 & $10 .-$ & 9.- & 9.25 & $9 .-$ & 12.50 & 9.75 & 10. \\
\hline $15-1-30$ & 10.50 & 11.- & 9.50 & 12.25 & 10.50 & 11.50 & $10 .-$ & 13.25 & 11.75 & 9.7 \\
\hline $3-2-30$ & 11.75 & 10.75 & 9.25 & 10.75 & 11.- & 11.25 & 11.25 & 12.25 & 12.25 & 10. \\
\hline
\end{tabular}

fication de la microméthode de Pregl d'après Parnas-Wagner. Le résidu sec et le résidu sec dégraissé ont été établis par le calcul.

\section{$3^{\circ}$ RECHERCHES PERSONNELLES.}

\section{A. Rendement laitier}

Plusieurs auteurs sont d'accord que la castration augmente la quantité de lait sécrétée $(2,19,22)$. Nos essais donnent le tableau suivant, en ce qui concerne la quantité de lait séerétée par les six vaches castrées, par dix vaches de même race traitées de façon identique, dans la même étable et la traite moyenne de 120 vaches dans la même étable. Le tableau I et le graphique correspondant montrent que la production des vaches castrées, d'abord inférieure de 0,43 litres à celle des vaches témoins, est, après 10 jours, supérieure à celle des témoins ; elle se maintient, à deux petites exceptions près, pendant 11 mois à ce niveau. Le graphique montre que les variations de la 


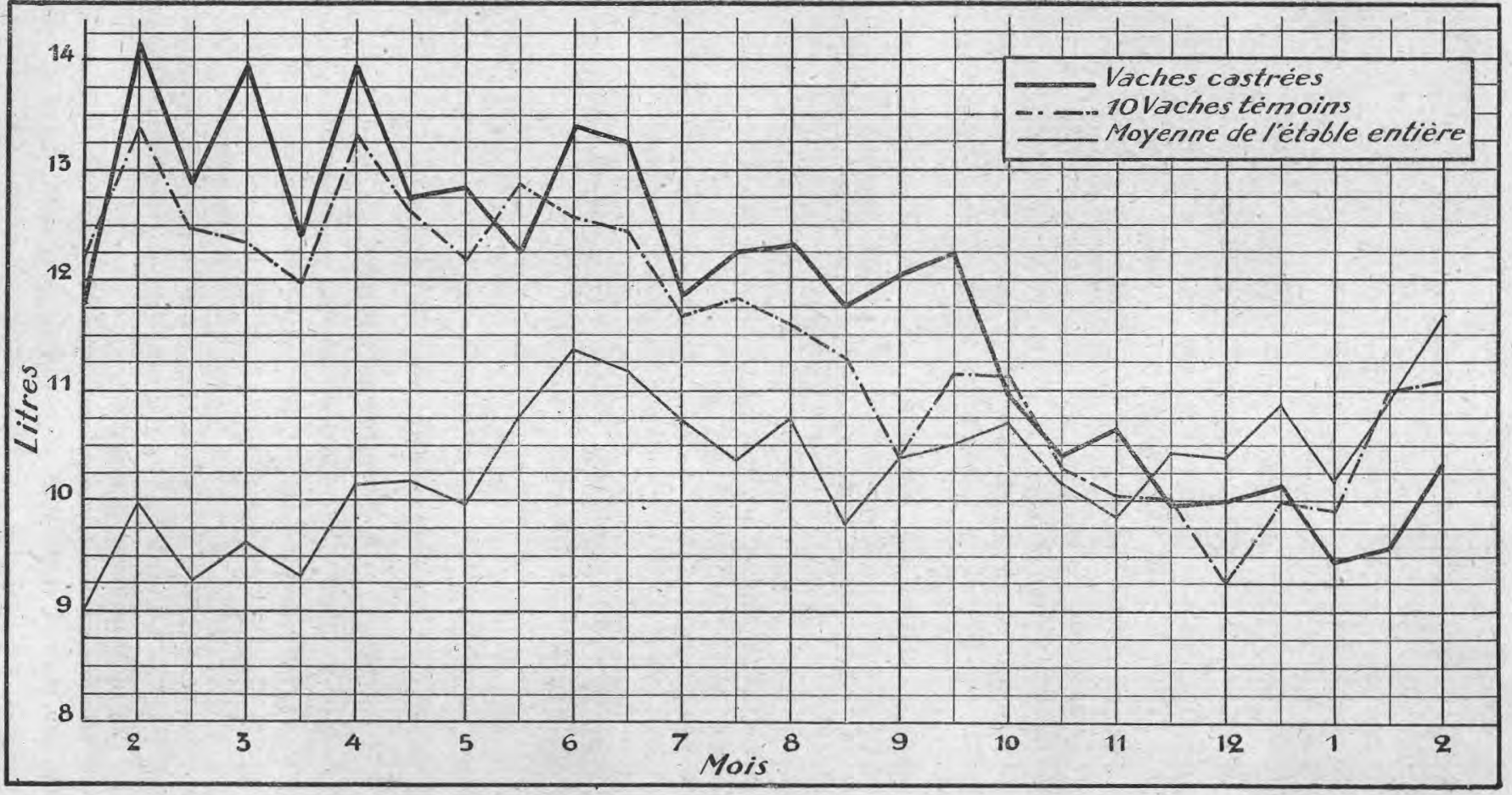


quantité de lait fournie par les vaches castrées sont à peu près parallèles à celles des témoins et de la traite moyenne de l'étable.

Pendant la durée des expériences, soit du $1^{\text {er }}$ février 1929 au 1 er février 1930, les vaches castrées ont donné une moyenne journalière de 11 litres 83 , Jes témoins 11 litres 41 , soit 0 litre 42 en moins.

Si l'on déduit du tableau I la différence moyenne journalière (0 litre 339) pendant la période du 1-II-1929 au 1-II-1930), on observera que les vaches castrées ont donné en moyenne 123 litres 7 de plus que les témoins.

\section{B. Composants du lait.}

10 Taux butyreux (en \%). - L'élévation du taux butyreux du lait par la castration n'est pour ainsi dire plus contestée. D'après de nombreux observateurs anciens $(1,9,10,14)$ et modernes $(3,5,11,16)$, français ou autres, l'augmentation du taux butyreux serəit très importante $(25-30 \%)$. Nos essais ont donné les résultats suivants :

TABLEAU II.

MATIERE GRASSE EN \% DANS LE LAIT (GERBER).

\begin{tabular}{|c|c|c|c|c|c|c|c|c|c|c|c|}
\hline Date de bessai & $8-I I I \mid$ & $12-\mathrm{III}$ & 14-III & $18-\mathrm{III}$ & $20-\mathrm{III}$ & 25-III & 28-III & $17-I V$ & $30-\mathrm{VII}$ & 1 -VIII & Moyenne \\
\hline Vaches castrées... & 3,38 & 3,58 & 3,31 & 3,09 & 3,59 & 3,31 & 3,10 & 3,48 & 3,21 & 3,28 & 3,33 \\
\hline Vaches témoins & 3,10 & 2,50 & 2,90 & 3,00 & 3,25 & 2,85 & 2,55 & 2,98 & 3,40 & 3,20 & 2,99 \\
\hline $\begin{array}{l}\text { Lait mélange éta } \\
\text { ble } \ldots \ldots \ldots \ldots \ldots\end{array}$ & 3,10 & - & 3,00 & 3,30 & 3,60 & 3,90 & 3,30 & 2,95 & 2,80 & 2,70 & 3,18 \\
\hline
\end{tabular}

En réalité, les vaches castrées donnent bien un Jait plus riche en matière grasse que les vaches témoins, mais cette augmentation diffère notamment de celle annoncée dans les travaux français : elle n'est que de $10 \%$ par rapport au lait des témoins et $4,7 \%$ par rapport au lait mélangé de l'étable.

$2^{\circ}$ Quantité de matière grasse en grammes. - A été déduite de la quantité de lait fournie au jour de l'essai.

TABLEAU III.

QUANTITÉ DE LATT AU JOUR DES ESSAIS EN LITRES.

\begin{tabular}{|c|c|c|c|c|c|c|c|c|c|c|c|}
\hline Date & $8-\mathrm{III}$ & 12-III & I4-III & $18-I I I$ & $20-\mathrm{III}$ & $\mid 25-\mathrm{III}$ & $28-\mathrm{III}$ & $17-I V$ & $30-\mathrm{VII}$ & 1-VII. & Moyenne \\
\hline Castrées .. & 14,50 & 12,85 & 13,56 & $\mid 14,56$ & 13,81 & 12,00 & $\mid 14,12$ & 12,06 & 14,44 & 11,67 & 13,36 \\
\hline Témoins .. & 10,00 & 17,25 & 13,13 & 12,88 & 12,88 & 12,75 & 12,38 & 12,25 & 12,50 & 12,88 & 12,89 \\
\hline Lait mél. . . & 9,35 & - & 9,21 & 9,42 & 9,42 & 10,27 & 10,27 & 10,10 & 9,72 & 9,31 & 9,67 \\
\hline
\end{tabular}


TABLEAU IV.

QUANTITÉ DE MATIK̀RE GRASSE EN GRAMMES.

\begin{tabular}{c|c|c|c|c|c|c|c|c|c|c|c|c|c|c}
\hline \hline Date & -III & 12-III & 14-III & 18-III & 20-III & $25-$ III & 28-III & 17I-V & 30-VI & 1-VIII & Moyenne \\
\hline Castrées .. & 492 & 413 & 450 & 413 & 494 & 389 & 439 & 412 & 449 & 382 & 433 \\
Témoins .. & 280 & 431 & 371 & 391 & 411 & 363 & 326 & 367 & 431 & 397 & 397 \\
Lait mél... & 390 & - & 276 & 311 & 339 & 401 & 339 & 298 & 272 & 251 & 320 \\
\hline \hline
\end{tabular}

A la faible augmentation de la quantité de lait et du taux butyreux correspond une augmentation de la quantité absolue de matière grasse, ne dépassant pas $10 \%$.

$3^{\circ}$ Lactose. - Selon Lermat, la castration provoquerait une augmentation telle du taux en lactose quelle serait déjà sensible au goût, et correspondrait à une valeur nutritive plus élevée du lait. V. L. J. Gouvan aurait fait des observations dans le même sens, tandis que CoRNevin aurait trouvé exactement le contraire. Nos propres essais infirmeraient plutôt les observations de LERMAT et de Golvan et confirmeraient celles de Cornevin.

TABLEAU $V$.

LACTOSE (SELON KOLTHOFF).

\begin{tabular}{c|c|c|c|c|c|c|c|c|c|c|c|c}
\hline \hline Date & 8-III & 12 -III & $14-\mathrm{III}$ & $18-\mathrm{III}$ & 20 -III & $25-\mathrm{III}$ & $28-\mathrm{III}$ & $17-\mathrm{IV}$ & 30 -VII & 1 -VIII & Moyenne \\
\hline & & & & & & & & & & & \\
Castrées .. & 6,00 & 5,54 & 5,49 & 7,65 & 4,59 & 3,69 & 5,49 & 5,40 & 5,70 & 4,98 & 5,45 \\
Témoins .. & 5,76 & 6,48 & 5,76 & 7,56 & 4,32 & 3,42 & 5,40 & 5,40 & 5,94 & 5,09 & 5,62 \\
Lait melangé & 5,76 & - & 5,40 & 6,84 & 4,68 & 3,24 & 5,76 & 5,40 & 5,40 & 5,04 & 5,50 \\
\hline \hline
\end{tabular}

La diminution est trop faible (3\%) et est comprise du reste dans les limites des variations normales pour qu'on puisse parler d'une modification appréciable.

$4^{\circ}$ Substances azotées. - A. D'après Marchand (14) et Menard (16), les taux en caséine, albumine et globuline seraient augmentés par la castration au point que le rendement en fromage serait de $30 \%$ supérieur, ce qui signifierait une élévation de la teneur en caséine. Lermat (11) observe aussi une augmentation certaine bien que variable. Nos recherches ne confirment pas ces observations.

Les taux en caséine, albumine et globuline ne sont pas augmentés, on observerait plutôt une très minime diminution de ces 3 substances azotées dans le lait des vaches castrées.

$5^{\circ}$ Reste d'azote. - La teneur en azote soluble dans l'alcool méthylique qui, en principe, pourrait être modifiée dans le lait des vaches 
TABLEAU VI.

CASEINE.

\begin{tabular}{|c|c|c|c|c|c|c|c|c|c|c|}
\hline Date & $12-\mathrm{III}$ & 14 -III & $18-\mathrm{III}$ & $20-$ III & $25-$ III & $28-I I I$ & 17-VII & 30 -VII & I-VIII & Moyenne \\
\hline Castrées.. & 4,704 & 3,508 & 3,770 & 3,636 & 3,770 & 3,788 & 3,272 & 3,794 & 3,788 & 3,764 \\
\hline Témoins . & 4,182 & 3,691 & 3,794 & 3,533 & 3,794 & 3,715 & 3,399 & 4,055 & 4,383 & 3,839 \\
\hline
\end{tabular}

ALBUMINE.

Castrées........
Témoins ......

GLOBULTNE.

\begin{tabular}{ll|l|l|l|l|l|l|l|l|l} 
Castrées $\ldots \ldots$ & $\ldots, 0,0,0$ \\
Témoins $\ldots .$. & - & 0,035 & 0,022 & 0,010 & 0,009 & 0,033 & 0,018 & 0,015 & 0,014 & 0,018 \\
0,025 & 0,029 & 0,009 & 0,008 & 0,050 & 0,032 & 0,014 & 0,012 & 0,030
\end{tabular}

castrées, a été déterminée par la méthode de ZARIBNICKY-MUNCHBERG. Le tableau VII montre qu'à ce point de vue le lait des vaches eastrées

TABLEAU VII.

AZOTE TOTAT.

\begin{tabular}{|c|c|c|c|c|c|c|c|c|c|c|c|}
\hline Date & 8-III & I2-III & 14-III & 18-III & 20-III & 25-III & 28-III & $17-I V$ & 30 -VII & 1-VIII & Moyenne \\
\hline Castrées ..... & $0,553 \%$ & 0.6203 & 0,5550 & 0,6235 & 0,5432 & 0,5674 & 0,5538 & 0,6302 & 0,5478 & 0,5146 & 0,5709 \\
\hline Témoins ...... & 0,5527 & 0,6934 & 0,5586 & 0,6094 & 0,5324 & 0,5912 & 0,5583 & 0,5954 & 0,5835 & 0,5380 & 0,5813 \\
\hline Etable ...... & 0,5376 & - & 0,6164 & 0,5954 & 0,5954 & 0,6052 & 0,5604 & 0,5688 & 0,5463 & 0,4903 & 0,5684 \\
\hline
\end{tabular}

ne présente aucune différence appréciable avec le lait des témoins ou le lait mélangé des 120 vaches.

\section{TABLEAU VIII.}

RESTE D'AZOTE (ZARIBNICKY-MÜNCHBERG).

\begin{tabular}{|c|c|c|c|c|c|c|c|c|c|c|c|}
\hline Date & 8-III & 12-III & 14-III & 18-III & 20-III & $25-\mathrm{III}$ & 28-III & $17-I V$ & 30 -VII & 1 -VIII & Moyenne \\
\hline Castrées & 0,0744 & 0,0637 & 0,0651 & 0,0455 & 0,0764 & 0,0497 & 0,0431 & 0,0859 & 0,0613 & 0,0584 & 0,0624 \\
\hline Témoins & 0,0816 & 0,0891 & 0,0545 & 0,0490 & 0,0911 & 0,0420 & 0,0490 & 0,0722 & 0,0624 & 0,0730 & 0,0664 \\
\hline Etable ....... & - & - & 0,0560 & 0,0532 & 0,0659 & 0,0659 & 0,0392 & 0,0556 & 0,0701 & 0,0560 & 0,0577 \\
\hline
\end{tabular}

60 Chlore. - La teneur en chlore du lait serait influencée par la castration soit en plus, soit en moins selon les auteurs. Le tableau. IX montre que le taux du chlore serait légèrement augmenté dans le lait des vaches castrées, mais le nombre des observations est trop restreint pour tirer une conclusion certaine. 
TABLEAU IX.

CHLORE.

\begin{tabular}{c|c|c|c|c|c|c|c|c|c|c|c|c|c|c|c}
\hline \hline Date & 8-III & 12-III & 14-III & 18-III & 20 -III & 25-III & 28 -III & 17-IV & 30 -VII & 1-VIII & Moyenne \\
\hline Castrées ..... & 0,08746 & 0,14068 & 0,09266 & 0,09842 & 0,10990 & 0,08864 & 0,13830 & 0,16040 & 0,14875 & 0,06626 & 0,11315 \\
Témoins ..... & 0,07092 & 0,12362 & 0,07802 & 0,12594 & 0,09934 & 0,08510 & 0,12058 & 0,14320 & 0,12991 & 0,08510 & 0,10617 \\
Etable ....... & 0,07092 & - & 0,11356 & 0,08748 & 0,08510 & 0,09930 & 0,14184 & 0,13474 & 0,12493 & 0,07092 & 0,10319 \\
\hline \hline
\end{tabular}

\section{Propriétés.}

10 Poids spécifique.

TABLEAU $\mathrm{X}$.

POIDS SPÉCIFIQUE (EN DEGRÉS DU LACTODENSIMÈTRE A $15^{\circ}$ ).

\begin{tabular}{c|c|c|c|c|c|c|c|c|c|c|c|c}
\hline \hline Date & 8 -III & 12 -III & 14-III & 18 -III & 20-III & 25 -III & 28 -III & $17-I V$ & 30 -VII & 1-VIII & Moyenne \\
\hline & & & & & & & & & & & \\
Castrées ... & 32,9 & 32,3 & 33,1 & 33,8 & 33,2 & 32,5 & 32,5 & 33,8 & 33,7 & 33,6 & 33,14 \\
Témoins ... & 32,4 & 32,3 & 33,4 & 33,9 & 32,9 & 33,4 & 32,6 & 34,3 & 33,3 & 33,7 & 33,23 \\
Etable ..... & 33,3 & - & 32,6 & 33,0 & 32,6 & 33,2 & 32,4 & 33,6 & 33,7 & 34,5 & 33,21 \\
\hline \hline
\end{tabular}

Comme il fallait s'y attendre d'après nos résultats antérieurs, la différence entre le poids spécifique du lait des vaches castrées et des témoins est peu importante. Le sens et l'intensité de cette différence correspondent au fait que le lait des vaches castrées est légèrement plus riche en matière grasse.

$2^{\circ}$ Point de congélation du lait. - Le glissement du point de congélation observé par différents auteurs français (11) ne se trouve pas confirmé par nos essais.

\section{TABLEAU XI.}

POINT DE CONGÉLATION DU LAIT.

\begin{tabular}{c|c|c|c|c|c|c|c|c|c|c|c|c|c}
\hline \hline Date & 8-III & 12 -III & 14 -III & 18 -III & 20 -III & 25 -III & 28 -III & $17-I V$ & 30 -VII & 1-VIII & Moyenne \\
\hline Castrées $\ldots$ & 5,59 & 5,51 & 5,56 & 5,53 & 5,66 & 5,77 & 5,67 & 5,76 & 5,60 & 5,62 & 5,63 \\
Témoins ... & 5,68 & 5,66 & 5,63 & 5,66 & 5,79 & 5,77 & 5,73 & 5,73 & 5,55 & 5,49 & 5,67 \\
Etable ..... & 5,42 & - & 5,50 & 5,57 & 5,73 & 5,83 & 5,71 & 5,70 & 5,61 & 5,60 & 5,63 \\
\hline \hline
\end{tabular}

\section{$3^{\circ}$ Degré d'acidité (Soxhlet et Henkel).}

En ce qui concerne le degré d'acidité, le lait des vaches castrées ne diffère pas sensiblement du lait des vaches témoins.

$4^{\circ}$ Coagulation par la présure. - Dans une capsule de porcelaine, 
TABLEAU XII.

DEGRÉ D'ACIDITÉ.

\begin{tabular}{c|c|c|c|c|c|c|c|c|c|c|c}
\hline Date & 8-III & 12-III & 14-III & 18-III & 20-III & 25-III & 28-III & 17-IV & 30 -VII & 1-VIII & Moyenne \\
\hline & & & & & & & & & & & \\
Castrées ... & 7,9 & 7,2 & 7,9 & 7,3 & 7,2 & 7,7 & 8,5 & 7,3 & 8,0 & 7,6 & 7,66 \\
Témoins ... & 7,8 & 7,8 & 8,0 & 7,3 & 6,8 & 8,4 & 8,5 & 8,0 & 8,7 & 8,5 & 7,98 \\
Etable.... & 7,7 & - & 7,6 & 7,2 & 7,0 & 7,8 & 8,4 & 8,0 & 8,2 & 7,2 & 7,66 \\
\hline \hline
\end{tabular}

dont l'intérieur est émaillé en couleur, on verse $50 \mathrm{~cm}$. de lait, $2 \mathrm{~cm}$. d'une solution de présure et on maintient le mélange à $35^{\circ}$. On détermine le temps de coagulation avec une chronomètre à répétition.

TABLEAU XIII.

COAGULABILITÉ PAR LA PRÉSURE.

\begin{tabular}{|c|c|c|c|c|c|c|c|c|c|c|c|}
\hline & & T & - & & & & & & & & Moyenne \\
\hline Castrées .. & 7'08', & 6'03" & 4'11', & $5^{\prime} 32^{\prime \prime}$ & 6'21"' & $5^{\prime} 18^{\prime \prime}$ & $3^{\prime} 00^{\prime \prime}$ & $2 ' 54^{\prime \prime}$ & $3^{\prime} 51^{\prime \prime}$ & 4'44", & $4^{\prime} 45^{\prime \prime}$ \\
\hline Témoins .. & 8'36"' & 4'50'” & 4'25'" & 2'56" & 6'01'" & $2^{\prime} 46^{\prime \prime}$ & 3'33" & $1^{\prime} 52^{\prime \prime}$ & 3'20', & 4'53" & 4'19'" \\
\hline Etable .... & 7'00'" & F & 6'30'" & 5'30' & 6'04' & 6'50', & $4^{\prime} 54^{\prime \prime}$ & $1^{\prime} 25^{\prime \prime}$ & $3^{\prime} 59^{\prime \prime}$ & $5^{\prime} 24^{\prime \prime}$ & $5^{\prime} 17^{\prime \prime}$ \\
\hline
\end{tabular}

On ne constate done aucune différence appréciable d'action de la présure sur le lait des vaches castrées et des témoins.

\section{Valeurs calculées.}

10 Résidu sec. - Le résidu sec du lait des vaches castrées (calculé au moyen de la formule de FLEISCHMANN à partir du poids spécifique et du taux butyreux) est en moyenne de 0,43 supérieur à celui du lait des témoins et $0,15 \%$ par rapport au lait mélangé des 120 vaches de l'étable. Les différences sont donc très faibles.

TABLEAU XIV,

RÉSIDU SEC.

\begin{tabular}{|c|c|c|c|c|c|c|c|c|c|c|c|}
\hline Date & 8-III & 12-III & 14-III & |18-III & 20-III & 25-III & 28-III & 17-IV & 30 -VII & 1-VIII & Moyenne \\
\hline Castrées & 12,449 & 12,354 & 12,514 & 12,706 & 12,586 & 12,351 & 12,096 & 12,889 & 12,543 & 12,603 & 12,536 \\
\hline Témoins ...... & 12,072 & 11,339 & 12,091 & 12,338 & 12,376 & 12,021 & 11,474 & 12,396 & 12,681 & 12,541 & 12,133 \\
\hline Etable ....... & 12,308 & - & 12,014 & 12,474 & 12,734 & 13,243 & 12,324 & 12,203 & 12,048 & 12,128 & 12,386 \\
\hline
\end{tabular}

$2^{\circ}$ Résidu sec dêgraissé. - Le résidu sec dégraissé du lait des vaches castrées est égal à celui des témoins. Cette constatation apporte une bonne confirmation de concordance des valeurs obtenues pour le lactose et le point de congélation qui peut en quelque sorte servir de mesure à la proportion des éléments dispersés en ions. 
TABLEAU XV.

RESIDU SEC DÉGRAISSÉ.

\begin{tabular}{|c|c|c|c|c|c|c|c|c|c|c|c|}
\hline Date & 8-III & $12-I I I$ & I 14 -III & $18-\mathrm{III}$ & $20-\mathrm{III}$ & $25-\mathrm{III}$ & $28-I I I$ & $17-I V$ & $30-\mathrm{VII}$ & $1-$ VIII & Moyenne \\
\hline Castrées .. & 9,065 & 9,004 & 9,201 & 9,118 & 9,269 & 9,038 & 8,996 & 9,414 & 9,330 & 9,320 & 9,176 \\
\hline Témoins . . & 8,972 & 8,839 & 9,194 & 9,338 & 9,126 & 9,171 & 8,924 & 9,421 & 9,281 & 9,341 & 9,161 \\
\hline Etable.... & 9,208 & - & 9,014 & 9,174 & 9,134 & 9,343 & 9,024 & 9,253 & 9,248 & 9,428 & 9,183 \\
\hline
\end{tabular}

CONCLUSIONS.

Des recherches effectuées de février 1929 à février 1930 sur le lait de 6 vaches castrées, de 10 vaches d'âge et de race identiques, servant de témoins, traitées et nourries de la même façon, ont montré, en ce qui concerne la quantité de lait secrétée, les variations de certains composants et de certaines propriétés que :

10 La quantité de lait fournie par les vaches castrées est de 123,7 litres supérieure à celle des vaches témoins.

$2^{\circ}$ Le taux butyreux, le poids absolu de matière grasse du lait des vaches castrées sont de $10 \%$ supérieurs à ceux du lait des témoins.

Le poids spécifique du lait des vaches eastrées subit dono une modification de sens et d'intensité correspondants.

$3^{\circ}$ Le lactose, les substances azotées, le point de congélation, la coagulabilité par la présure, le résidu sec diffèrent peu dans le lait des vaches castrées ou des témoins.

Cependant, la teneur en chlore du lait des vaches castrées semble subir une légère augmentation. Ce fait doit être confirmé par des recherehes plus étendues.

\section{BIBLIOGRAPHIE}

[1] Dieulafait, Journ. d'agric. prat. 28, 1864.

[2] FALK, Berl. Tierâratl. Wochenschr., 1901, S. 333.

[3] Foges A., Wiener Klin. Wochenschr. 137, 1908.

[4] Frenzeu, Intelligenzblatt, Leipzig, 1759, No 52, Art. 7. cit. in Martiny, 1. c. S. 241.

[5] Gouvan V. L. J., La castration des vaches laitières, Hennobont, 1924.

[6] Gruter F., Schweizer, Archiv f. Tierheilkunde, 66, 48, 1924.

[7] Hintermann K., Der Pionnier, 6, Heft 21, S. 10, 1929.

[8] Коцтношт J. M., Zeitsch. f. Unters.. u. Nahrungs. Genussmittel, 45, 45, 923.

[9] Lebland, cit. in Spann 1. c. J. 2.

[10] Lejoux H. ref. Ohem. Centralblatt 1890, 586.

[11] LeRmat H., L'ovariotomie des vaches laitières, Editions médicales, Paris, 1924.

[12] Levrat, eit. in Golvan, 1. c.

[13] LӧнR, L., Fortschritte d. Landwirtschaft, 3, 681, 1928. 
[14] Marohand eit. in Martiny, 1. c. S. 243.

[15] Mantivy B., Die Milch, ihr Wesen und ihre Ververtung, Danzig, A. W. Kafemann, 1871.

[16] Ménard, Extrait d'une lettre de Ménard, Annal. de l'agric. franc., 1857, S. 288.

[17] Möвгоs P. J., Uber die Wirkung der Kastration, Beitrag zur Lehre von den Geschlechtsunterschieden, Heft 3/4, C. Marhold, Leipzig 1903, S. 37.

[18] MüNCHBerg F., Milchw. Forschungen, 3, 404, 1926.

[19[ NuEsch, Berl., Tierarztl. Wochenschr. 1910, No 47.

[20] Retzios, Om Kastration af Kor, Stockholm 1835, S. 6, cit. in Martiny, 1. c. S. 241.

[21] Rossmeiser J., Biochem. Zeitschr., 16, 164, 1909.

[22] Spann, Landw. Fachpresse f. d. Tschechoslowakei, 8, 1, 1930.

[23] WINN, Recueil de médec. vétér. prat, 1834 cit. in Martiny, 1. e. S. 241.

[24a] ZARIBNICKY F., Milchw. Forschungen 3, 404, 1926.

[24b] ZaRIBNIoKY F., Wiener, Tierärztl. Monatschrift, 14, 450, 1927.

\title{
OBSERVATIONS PRATIQUES SUR LE CALCUL DU RENDEMENT EN BEURRERIE
}

\author{
par M. E. VAILLANT, \\ Ingénieur agronome
}

(Fin)

\section{DISCUSSION ET INTERPRÉTATION DU CALCUL DE RENDEMENT.}

Afin de nous permettre de tirer de ces ealculs tous les enseignements qu'ils comportent, nous reproduisons ci-dessous la copie d'une page du livre de fabrication d'une usine où cette méthode est a ppliquée. Armé de ce document, nous allons l'étudier en détail et tirer, de tous les chiffres qui y sont consignés, les commentaires, constatations et critiques qu'ils a ppellent.

Avant de prendre contact avec ce tableau, nous croyons utile de situer exactement l'usine et le travail qui s'y fait.

La laiterie qui nous intéresse est installée dans un important centre d'élevage de vaches hollandaises (ce qui explique la faible teneur en matière grasse du lait à cette époque de l'année). Cette laiterie, qui ramasse en moyenne 10.000 litres par jour en hiver et 18.000 litres en été, livre à la consommation une moyenne journalière assez régulière de 5.500 à 6.000 litres. Lo reste est travaillé à l'usine pour être écrémé. Le beurre obtenu est en partie mis en pains et vendu à la ville voisine, l'autre partie est expédiée en mottes de $10 \mathrm{~kg}$. ou en caisses de $25 \mathrm{~kg}$.

Lo lait écrémé, a près distribution aux producteurs de la quantité 